PROCEEDINGS OF THE

AMERICAN MATHEMATICAL SOCIETY

Volume 130, Number 5, Pages 1339-1343

S 0002-9939(01)06312-2

Article electronically published on December 20, 2001

\title{
HÖLDER ESTIMATES OF SOLUTIONS TO A DEGENERATE DIFFUSION EQUATION
}

\author{
YUNGUANG LU
}

(Communicated by Suncica Canic)

\begin{abstract}
This paper is concerned with the Hölder estimates of weak solutions of the Cauchy problem for the general degenerate parabolic equations

$$
u_{t}=\Delta G(u)+\sum_{j=1}^{N} f_{j}(u)_{x_{j}}+h(u)
$$

with the initial data $u(x, 0)=u_{0}\left(x_{1}, x_{2}, \ldots, x_{N}\right)$, where the diffusion function $G(u)$ can be a constant on a nonzero measure set, such as the equations of two-phase Stefan type. Some explicit Hölder exponents of the composition function $G(u)$ with respect to the space variables are obtained by using the maximum principle.
\end{abstract}

This paper is concerned with the Hölder estimates of solutions of the Cauchy problem for the general degenerate parabolic equations

$$
u_{t}=\Delta G(u)+\sum_{j=1}^{N} f_{j}(u)_{x_{j}}+h(u),
$$

with initial data

$$
u(x, 0)=u_{0}\left(x_{1}, x_{2}, \ldots, x_{N}\right),
$$

where $G(u)$ is a nondecreasing smooth function, and $N$ denotes the space dimension. If $G(u) \equiv$ const and one of the $f_{j}(u)$ is nonlinear, (1) is the standard nonlinear hyperbolic conservation law with a source. In this case, the continuous solution of (1), in general, exists only locally in time. This means that shock waves appear in the solution in finite time.

It is well-known that degenerate parabolic equations do not possess classical solutions. When $g(u)=G^{\prime}(u)=0$ at some points, the solution $u$ of the Cauchy problem (1) and (2), in general, fails to be smooth at the interface between a parabolic region and a region of parabolic degeneracy. Fortunately, if the diffusion function $G(u)$ has only one degenerate point, such as the porous media equation, i.e., $G(u)=u^{m}(m>1)$, the regularity of weak solutions can be proved. We refer to the references in the survey paper [9] and the most recent papers [4, 8, 10, 11, 12, 14] for the details.

If $G(u)$ is degenerate on an interval, for instance the two-phase Stefan problem, the continuity of $G(u)$ was first proved in [3], and the Hölder continuity of $G(u)$

Received by the editors April 12, 2000.

2000 Mathematics Subject Classification. 35K55, 35K65, 35D10, 35K15.

Key words and phrases. Degenerate parabolic equation, Hölder solution, maximum principle. 
with an implicit Hölder exponent was obtained in [6]. Some special cases for $G(u)$ having a degenerate interval were also studied in [13].

In this paper, we study the Hölder continuity of solutions, in $R^{N} \times[0, T]$ for any given time $T$, for the Cauchy problem $(1),(2)$ in more general functions $G(u), f_{j}(u)$ and $h(u)$.

The main results in this paper are given in the following theorem:

Theorem 1. Suppose that $G^{\prime}(u) \geq 0$ and the solution $u$ is bounded: $|u| \leq \bar{u}$. If

$$
\left|\frac{G G^{\prime \prime}}{G^{\prime 2}}\right| \leq \beta, \quad \beta^{2} \leq \frac{1}{2 N}
$$

and

$$
\left|f_{j}^{\prime \prime}(u)\right| \leq c\left|G^{\prime \prime}(u)\right|, \quad\left|h^{\prime}(u)\right| \leq c G^{\prime}(u)
$$

for a suitable positive constant $c$, then the solutions u of the Cauchy problem (1),(2) satisfy the global estimates

$$
\left|\nabla G^{\alpha}(u)\right| \leq M \quad \text { on } \quad R^{N} \times R^{+}
$$

if the initial data (2) satisfies the same regular estimate (5), where $M$ is a positive constant depending only on the initial data, and

$$
\alpha>1-\frac{1+\sqrt{1-2 N \beta^{2}}}{4} .
$$

The equality in (6) is true if $h(u) \equiv 0$ and $f_{j}(u) \equiv 0$.

Proof of Theorem 1. Without loss of generality, let $G(u) \geq 0$. We consider the Cauchy problem for an equation related to (1),

$$
u_{t}=\Delta G^{\epsilon}(u)+\sum_{j=1}^{N} f_{j}(u)_{x_{j}}+h(u)
$$

with the initial data

$$
u^{\epsilon}(x, 0)=u_{0}\left(x_{1}, x_{2}, \ldots, x_{N}\right)+\epsilon_{1},
$$

where $G^{\epsilon}(u)=G(u)+\epsilon(u+\bar{u})$, with $\epsilon$ and $\epsilon_{1}$ small nonnegative constants. For the porous medium equation, we choose $\epsilon=0, \epsilon_{1}>0$; for other cases, we choose $\epsilon_{1}=0, \epsilon>0$. Thus for fixed $\epsilon$ and $\epsilon_{1}$, the equation (7) is strictly parabolic. We can resolve the Cauchy problem (7), (8) and obtain the approximate solutions $u^{\epsilon, \epsilon_{1}}$. Since we assume $u^{\epsilon, \epsilon_{1}}$ to be bounded, there exists a subsequence $u^{\epsilon^{l}, \epsilon_{1}^{l}}$ converging weakly to a bounded function $u$ as $\epsilon^{l}, \epsilon_{1}^{l} \rightarrow 0^{+}$, on any bounded region. If we can prove the Hölder continuity of $G\left(u^{\epsilon, \epsilon_{1}}\right)$, then $G\left(u^{\epsilon^{l}, \epsilon_{1}^{l}}\right)$ converges weakly to $G(u)$ by applying the Minty device with the condition $G^{\prime}(u) \geq 0$. From the conditions in (4), we can prove that $f_{j}\left(u^{\epsilon^{l}, \epsilon_{1}^{l}}\right)$ and $h\left(u^{\epsilon^{l}, \epsilon_{1}^{l}}\right)$ converge weakly to $f_{j}(u)$ and $h(u)$ respectively. Therefore the limit $u$ is a weak solution of the Cauchy problem (1) and (2), and $G(u)$ satisfies the same Hölder estimates. This technique is standard, and the details can be found in 12 . Here we only give the proof of the uniform estimate (5).

Make the transformation

$$
w=\frac{1}{2} \sum_{i=1}^{N}\left(G^{\epsilon}(u)_{x_{i}}\right)^{2}
$$


and set $P(u)=G^{\epsilon}(u)$. From (7), we obtain

$$
P_{t}=P^{\prime}(u) \Delta P+\sum_{j=1}^{N} f_{j}^{\prime}(u) P_{x_{j}}+P^{\prime}(u) h(u),
$$

$$
\left(P_{x_{i}}\right)_{t}=P^{\prime}(u) \Delta\left(P_{x_{i}}\right)+\frac{P^{\prime \prime}(u)}{P^{\prime}(u)} P_{x_{i}} \Delta P+\left(\sum_{j=1}^{N} f_{j}^{\prime}(u) P_{x_{j}}\right)_{x_{i}}+\left(P^{\prime}(u) h(u)\right)_{x_{i}}
$$

and

$$
\begin{aligned}
w_{t}= & P^{\prime}(u) \Delta w-\sum_{i, j=1}^{N} P^{\prime}(u)\left(P_{x_{i} x_{j}}\right)^{2}+\frac{2 P^{\prime \prime}(u)}{P^{\prime}(u)} w \Delta P+\sum_{j=1}^{N} f_{j}^{\prime}(u) w_{x_{j}} \\
& +\sum_{j=1}^{N} \frac{2 f_{j}^{\prime \prime}(u)}{P^{\prime}(u)} P_{x_{j}} w+2\left(\frac{P^{\prime \prime}(u) h(u)}{P^{\prime}(u)}+h^{\prime}(u)\right) w .
\end{aligned}
$$

Let $z=P^{s} w$, with $s$ a constant. By simple calculations,

$$
\begin{aligned}
\Delta w= & \Delta\left(P^{-s} z\right)=\sum_{j=1}^{N}\left(P^{-s} z\right)_{x_{j} x_{j}} \\
= & \sum_{j=1}^{N}\left[\left(P^{-s}\right)_{x_{j} x_{j}} z+2\left(P^{-s}\right)_{x_{j}} z_{x_{j}}+P^{-s} z_{x_{j} x_{j}}\right] \\
= & \sum_{j=1}^{N}\left[\left(-s P^{-s-1} P_{x_{j} x_{j}}+s(s+1) P^{-s-2} P_{x_{j}}^{2}\right) z\right. \\
= & \left(-s P^{-s-1} \Delta P+2 s\left(P^{-s}\right)_{x_{j}} z_{x_{j}}+P^{-s} z_{x_{j} x_{j}}\right] \\
& +\sum_{j=1}^{N}\left[-2 s P^{-s-1} P_{x_{j}} z_{x_{j}}+P^{-s} z_{x_{j} x_{j}}\right] .
\end{aligned}
$$

Then we have by (10)-(13) that

$$
\begin{aligned}
z_{t}= & P^{\prime}(u) \Delta z-2 s P^{-1} P^{\prime}(u) \sum_{i=1}^{N} P_{x_{i}} z_{x_{i}} \\
& +2 s(s+1) P^{-s-2} P^{\prime}(u) z^{2}-\sum_{i, j=1}^{N} P^{s} P^{\prime}(u)\left(P_{x_{i} x_{j}}\right)^{2}+\frac{2 P^{\prime \prime}(u)}{P^{\prime}(u)} z \Delta P \\
& +\sum_{j=1}^{N} f_{j}^{\prime}(u) z_{x_{j}}+\sum_{j=1}^{N} \frac{2 f_{j}^{\prime \prime}(u)}{P^{\prime}(u)} P_{x_{j}} z \\
& +\left(s P^{-1} P^{\prime}(u) h(u)+2 h^{\prime}(u)+\frac{2 P^{\prime \prime}(u) h(u)}{P^{\prime}(u)}\right) z
\end{aligned}
$$

Since

$$
\sum_{i, j=1}^{N}\left(P_{x_{i} x_{j}}\right)^{2} \geq \frac{1}{N}(\Delta P)^{2}
$$


we choose $s$ such that

$$
s(s+1)+\frac{N}{2}\left(\frac{P^{\prime \prime} P}{P^{\prime 2}}\right)^{2}<0 ;
$$

then $-1<s<0$ and there exists a suitable constant $C$ such that

$$
\begin{aligned}
& 2 s(s+1) P^{-s-2} P^{\prime}(u) z^{2}-\sum_{i, j=1}^{N} P^{s} P^{\prime}(u)\left(P_{x_{i} x_{j}}\right)^{2}+\frac{2 P^{\prime \prime}(u)}{P^{\prime}(u)} z \Delta P \\
& \quad \leq 2 s(s+1) P^{-s-2} P^{\prime}(u) z^{2}-\frac{1}{N} P^{s} P^{\prime}(u)(\Delta P)^{2}+\frac{2 P^{\prime \prime}(u)}{P^{\prime}(u)} z \Delta P \\
& \quad \leq-C P^{-s-2} P^{\prime}(u) z^{2} .
\end{aligned}
$$

From (14) and (17), we have

$$
\begin{aligned}
z_{t} \leq & P^{\prime}(u) \Delta z-2 s P^{-1} P^{\prime}(u) \sum_{i=1}^{N} P_{x_{i}} z_{x_{i}} \\
& -C P^{-s-2} P^{\prime}(u) z^{2}+\sum_{j=1}^{N} f_{j}^{\prime}(u) z_{x_{j}}+\sum_{j=1}^{N} \frac{2 f_{j}^{\prime \prime}(u)}{P^{\prime}(u)} P_{x_{j}} z \\
& +\left(s P^{-1} P^{\prime}(u) h(u)+2 h^{\prime}(u)+\frac{2 P^{\prime \prime}(u) h(u)}{P^{\prime}(u)}\right) z .
\end{aligned}
$$

If the conditions in Theorem 1 are satisfied, then there exist constants $c_{i}>0, i=$ $1,2,3,4,5$, such that

$$
\begin{aligned}
& \left|\sum_{j=1}^{N} \frac{2 f_{j}^{\prime \prime}(u)}{P^{\prime}(u)} P_{x_{j}} z\right| \leq 2 c \frac{\left|G^{\prime \prime}(u)\right|}{P^{\prime}(u)} \sum_{j=1}^{N}\left|P_{x_{j}}\right| z \\
& \quad \leq c_{1} \frac{\left|G^{\prime \prime}(u)\right|}{P^{\prime}(u)} w^{\frac{1}{2}} z=c_{1} \frac{P^{\frac{s}{2}+2}\left|G^{\prime \prime}(u)\right|}{P^{\prime}(u)^{2}} P^{-s-2} P^{\prime}(u) z^{\frac{3}{2}}
\end{aligned}
$$

where

$$
c_{1} \frac{P^{\frac{s}{2}+2}\left|G^{\prime \prime}(u)\right|}{P^{\prime}(u)^{2}} \leq c_{2} \frac{P(u)\left|G^{\prime \prime}(u)\right|}{P^{\prime}(u)^{2}} \leq c_{2} M(\epsilon),
$$

and $M(\epsilon) \rightarrow \beta$ as $\epsilon \rightarrow 0$. So $M(\epsilon)$ is uniformly bounded. Moreover,

$$
\begin{gathered}
\mid\left(s P^{-1} P^{\prime}(u) h(u) z|=| s P^{s+1} h(u)\left(P^{-s-2} P^{\prime}(u) z\right) \mid \leq c_{3} P^{-s-2} P^{\prime}(u) z,\right. \\
\left|2 h^{\prime}(u) z\right|=\left|\frac{2 h^{\prime}(u) P^{s+2}}{P^{\prime}(u)}\left(P^{-s-2} P^{\prime}(u) z\right)\right| \leq c_{4} P^{-s-2} P^{\prime}(u) z
\end{gathered}
$$

and

$$
\begin{aligned}
& \left.\mid \frac{2 P^{\prime \prime}(u) h(u)}{P^{\prime}(u)}\right) z|=| 2 P^{s+1} h(u)\left(\frac{P(u) G^{\prime \prime}(u)}{P^{\prime}(u)^{2}}\right)\left(P^{-s-2} P^{\prime}(u) z\right) \mid \\
& \quad \leq c_{5}\left(\frac{P(u)\left|G^{\prime \prime}(u)\right|}{P^{\prime}(u)^{2}}\right)\left(P^{-s-2} P^{\prime}(u) z\right) \leq c_{5} M(\epsilon)\left(P^{-s-2} P^{\prime}(u) z\right) .
\end{aligned}
$$

We have from (18)-(23) that

$$
\begin{aligned}
z_{t} \leq & P^{\prime}(u) \Delta z-2 s P^{-1} P^{\prime}(u) \sum_{i=1}^{N} P_{x_{i}} z_{x_{i}} \\
& +\left(-C z^{2}+c_{2} M(\epsilon) z^{\frac{3}{2}}+\left(c_{3}+c_{4}\right) z+c_{5} M(\epsilon) z\right) P^{-s-2} P^{\prime}(u),
\end{aligned}
$$


where $M(\epsilon)$ is uniformly bounded. Thus we have $z \leq M$ for a suitable large positive constant $M$ by using the maximum principle in (24).

From condition (3),

$$
\left(\frac{P^{\prime \prime} P}{P^{\prime 2}}\right)^{2}=\left(\frac{G^{\prime \prime}(u)(G(u)+\epsilon(u+\bar{u}))}{\left(G^{\prime}(u)+\epsilon\right)^{2}}\right)^{2} \leq \beta^{2}+\beta(\epsilon),
$$

where $\beta(\epsilon)>0$ is a function of $\epsilon$ and $\beta(\epsilon) \rightarrow 0$ as $\epsilon \rightarrow 0$. If we choose $s^{*}$ to be the smaller root of the equation

$$
s(s+1)+\frac{N}{2}\left(\beta^{2}+\beta(\epsilon)\right)=0,
$$

then (16) is satisfied. Thus we have

$$
P^{s^{*}} w \leq M
$$

where

$$
s^{*}=\frac{-1-\sqrt{1-2 N\left(\beta^{2}+\beta(\epsilon)\right)}}{2} .
$$

Letting $\epsilon \rightarrow 0$ in (28), we obtain the estimate (5), and with it the proof of Theorem 1.

\section{REFERENCES}

[1] S.B. Angenent and D.G. Aronson, The focusing problem for the radially symmetric porous medium equation, Commun. in P.D.E., 20(1995), 1217-1240. MR 96c:35074

[2] H. Brezis and M.G. Crandall, Uniqueness of solution of the initial value problem for $u_{t}-$ $\Delta G(u)=0$, J. Pure Appl. Math., 58(1979), 153-163. MR 80e:35029

[3] L.A. Caffarelli and L.C. Evans, Continuity of the temperature in the two-phase Stefan problem, Arch. Rat. Mech. Anal., 83(1983),199-220. MR 84g:35070

[4] L.A. Caffarelli, J.L. Vazquez and N.I. Wolanski, Lipschitz continuity of solutions and interfaces of the N-dimensional porous medium equation, Indiana Univ. Math. Journal, 36(1987), 373-401. MR 88k:35221

[5] E. DiBenedetto, Degenerate Parabolic Equations, Springer-Verlag, 1993 MR 94h:35130

[6] E. DiBenedetto and V. Vespri, On the Singular Equation $\beta(u)_{t}=\Delta u$, Arch. Rat. Mech. Anal., 132(1995), 247-309. MR 96m:35179

[7] A. Friedman, Partial Differential Equations of Parabolic Type, Englewood Cliffs,N.J.: Prentice-Hall Inc. 1964. MR 31:6062

[8] W. Jäger and Y.G. Lu, Global regularity of solutions for general degenerate parabolic equations in 1-D, J. Diff. Equs., 140(1997), 365-377. MR 98j:35112

[9] A.S. Kalashnikov, Some problems of the qualitative theory of non-linear degenerate secondorder parabolic equations, Russian Math. Surveys, 42, 169-222(1987). MR 88h:35054

[10] Y.G. Lu, Hölder Estimates of Solutions of Some Doubly Nonlinear Degenerate Parabolic Equations, Commun. in P.D.E., 24(1999), 895-914. MR 2000c:35105]

[11] Y.G. Lu, Hölder Estimates of Solutions of Biological Population Equations, Appl. Math. Letters, 13(2000), 123-126. MR 2001b:35182

[12] Y.G. Lu and W. Jäger, Regularity of Solutions to Nonlinear Reaction-Diffusion-Convection Equations with Degenerate Diffusion, J. Diff. Equations, 170(2001), No.1, 1-21. MR 2001j:35159

[13] Y. G. Lu, Hölder Estimates of Solutions for General Degenerate Parabolic Equation, to appear in Applicable Analysis.

[14] Y. G. Lu and L.W. Qian, Regularity of viscosity solutions of a degenerate parabolic equation, to appear in Proc. AMS.

Departamento de Matemáticas y Estadística, Universidad Nacional de Colombia, Bogotá, Colombia - and - Department of Mathematics, University of Science \& Technology of China, Hefei, People's Republic of China

E-mail address: yglu@matematicas.unal.edu.co 